\title{
Archéopages
}

Archéopages

Archéologie et société

Hors-série 3 | 2012

Nouveaux champs de la recherche archéologique

\section{Une connaissance de l'âge du Bronze transfigurée par l'archéologie préventive}

Patrice Brun et Cyril Marcigny

\section{OpenEdition}

1 Journals

Édition électronique

URL : https://journals.openedition.org/archeopages/701

DOI : 10.4000/archeopages.701

ISSN : 2269-9872

Éditeur

INRAP - Institut national de recherches archéologiques préventives

Édition imprimée

Date de publication : 1 janvier 2012

Pagination : 132-139

ISSN : 1622-8545

Référence électronique

Patrice Brun et Cyril Marcigny, « Une connaissance de l'âge du Bronze transfigurée par l'archéologie

préventive », Archéopages [En ligne], Hors-série 3 | 2012, mis en ligne le 01 janvier 2012, consulté le 24 février 2023. URL : http://journals.openedition.org/archeopages/701 ; DOI : https://doi.org/10.4000/ archeopages.701 

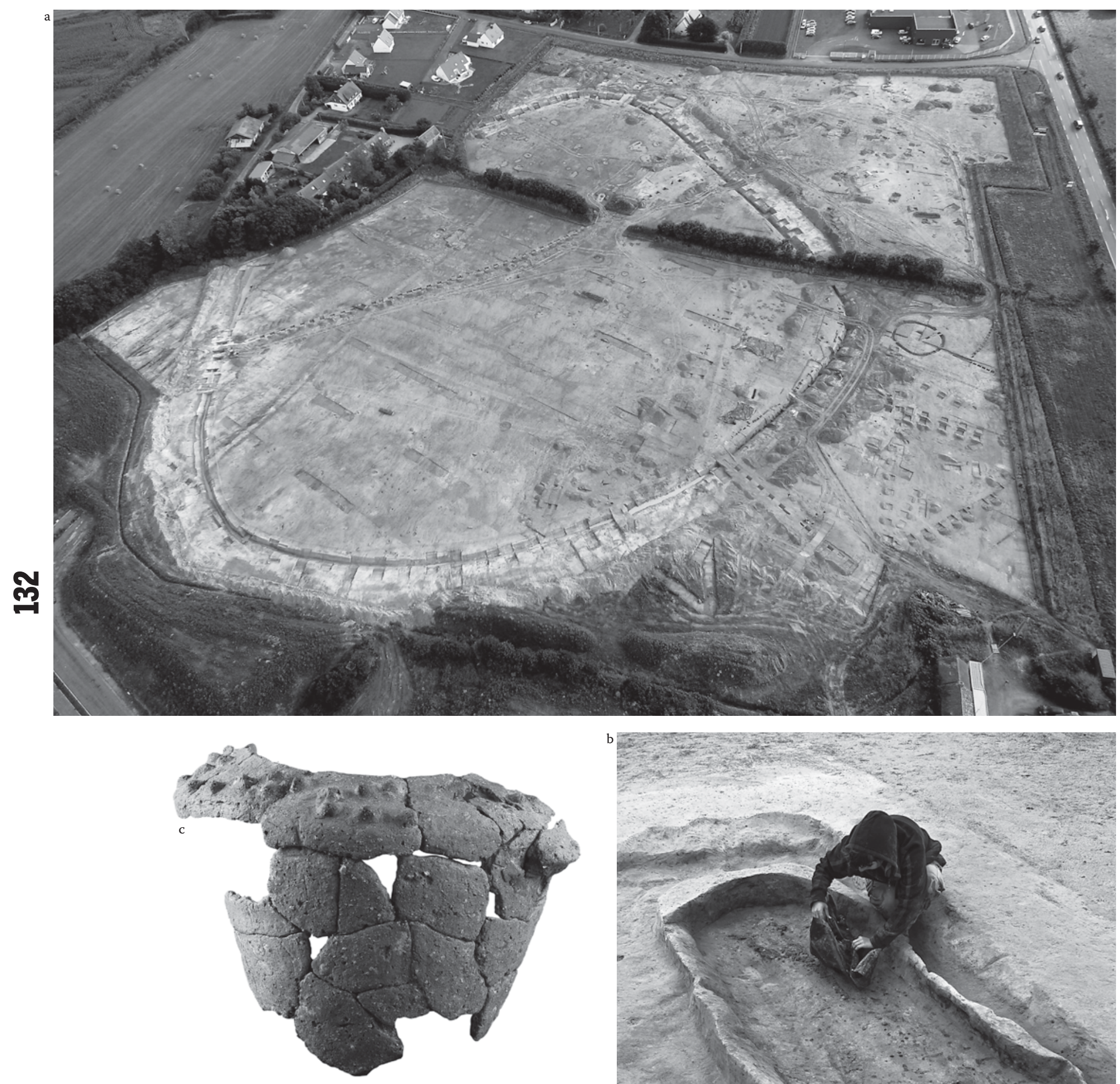

[Fig.1] À proximité d'un «Tumulus

armoricain » déjà repéré anciennement,

des fouilles préventives, conduites

sous la direction de Yoann Escats

(Inrap) à Lannion (Côtes-d'Armor),

ont permis la découverte d'une vaste

enceinte (a) ainsi que de deux autres.

monuments funéraires (b). L’ensemble

est daté de la fin du Bronze ancien ou

du début du Bronze moyen et associe,

pour la première fois, structure d'habita

et ensemble sépulcral. Le mobilier

céramique (c) recueilli dans les fossés

de l'enceinte est très proche de celui

connu dans le Centre-Ouest de la France.

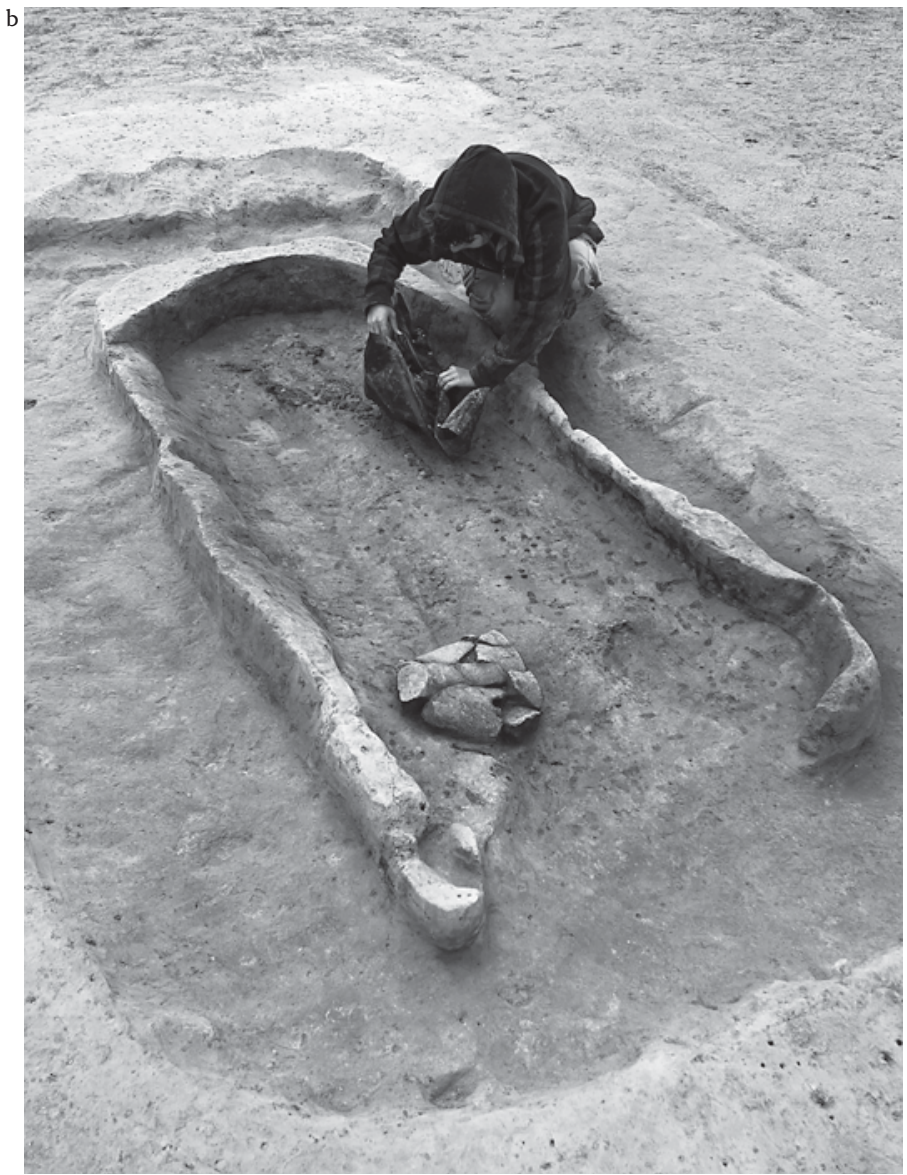


Pour cela, il est encore nécessaire d'étoffer le corpus de sites et de tenter de rééquilibrer les disparités régionales en mettant à profit une dynamique désormais bien affirmée.

BOSTYN F., SÉARA F., 2011 : Occupations de plein air mésolithique et néolithique : Le site de la Presle à Lhéry dans la Marne, S.P.F, Travaux 10, $287 \mathrm{p}$.

CLARK J.G.D., 1975 : The earlier stone age settlement of Scandinavia, Cambridge, Cambridge University Press, $282 \mathrm{p}$.

Ducroce T. 2001 : Le Mésolithique du Bassin de la Somme. Insertion dans un cadre morphostratigraphique, environnemental et culturel, publications du C.E.R.P., $n^{\circ} 7$, Lille, 253 p., 200 fig.

Ducrocq T., Bridault A., Coutard S., 2008 : « Le gisement mésolithique de Warluis (Oise) : approche préliminaire », in FAGNART J.-P. et al., p. 85-106.

DUCROCQ T., 2010 : "Quelques exemples dans le bassin hydrographique de la Somme ", in Le diagnostic des sites paléolithiques et mésolithiques, Actes du séminaire des 5-6 décembre 2006, Cahier de l'Inrap, $n^{\circ} 3$, p. $35-48$.

DUCROCQ T., à paraître : « Le Beuronien à segments dans le Nord de la France. Prémices d'une approche palethnologique », in VALENTIN B. et al.

Fagnart J.-P., Thévenin A., Ducrocq T., Souffi B., Coudret P. (Dir), 2008 : Le début du Mésolithique en Europe du Nord-Ouest, Actes de la table ronde d'Amiens, 9-10 octobre 2004, Paris, éd. Société préhistorique française (Mémoire XLV).

GUERET C., à paraître : «Identité et variabilité de l'outillage lithique du Premier Mésolithique en Belgique et dans le Nord de la France : Les apports de l'analyse fonctionnelle », in VALENTIN B.et al.

Hinout J., 1990 : «Évolution des cultures épipaléolithiques et mésolithiques dans le Bassin Parisien », Revue Archéologique de Picardie, ${ }^{\circ} 3 / 4$, p. 5-14.

KILDEA F., 2008a : « Les occupations du Mésolithique ancien et moyen de Saint-Romain-sur-Cher (Loir-et-Cher) », in FAgnarT J. P. et al., p. $153-167$.

KILDÉA F. (DIR.), 2008b : «La Croix de Bagneux » à Mareuil-sur-Cher (Loir-et-Cher) (A85, section $M_{3}$, site 30$)$. Un site paléolithique à occupations multiples dans la vallée du Cher, Rapport final d'opération de fouille, Inrap, $643 \mathrm{p}$.

LANG L., 1997: Occupations mésolithiques dans la moyenne vallée de la Seine. Rueil-Malmaison «Les Closeaux » (Hauts de Seine), Document Final de Synthèse, Afan A86, SRA Ile-de-France, 2 vol., 384 p.

LANG L., KILDÉA F., 2007: A85, K3, Ingrandes-de-Touraine, site F, « La prairie d'Ingrandes » (Indre-et-Loire), Rapport final l'opération de fouille, Inrap, $88 \mathrm{p}$.

LANG L., SICARD S., 2008 : « Les occupations mésolithiques des Closeaux à Rueil-Malmaison (Hauts-de-Seine) ", in FAGNART J. P. et al., p. 65-83.

Mordant C., Mordant D., 1989 : « Noyen-sur-Seine, site mésolithique en milieu humide fluviatile ", in L'homme et l'eau au temps de la Préhistoire, Actes du 112e congrès national des sociétés savantes, Lyon, 1987, p. 33-52.

Newell R., Kielman D., Constandse-Westermann T.S., Van Der SANDEN W.A.B., VAN GIJN A., 1990 : An inquiry into the ethnic resolution of mesolithic regional groups. The study of their decorative ornaments in time and space, Leiden, Brill $488 \mathrm{p}$.

Rozoy J.-G., 1978 : Les derniers chasseurs. L'Épipaléolithique en France et en Belgique, Mémoire de la Société d'Archéologie Champenoise, 3 vol., 1256 p., 294 fig, 259 pl., 81 tabl.

Rozoy J.-G., 1998 : « Stratégies de chasse et territoires tribaux au Mésolithique ", Bulletin de la Société Préhistorique Française, 95, 4, p. 525-536

SÉARA F., Rotillon S., Cupillard C. (DIR.), 2002 : Campement mésolithiques en Bresse jurassienne; Choisey et Ruffey-sur-Seille (Jura), Paris, MSH (DAF, 92), 344 p.

SÉARA F., 2006 : « Elements of reflection about habitat structural parts from spatial analysis of open air encampments in eastern France : The examples of Ruffey-sur-seille and Choisey (Jura) and Pont-surYonne (Yonne) », in Claus-JoAChim K. (ÉD.), After the Ice Age, Rottenburg, p. 277-283, 3 fig.

SÉARA F., 2008 : Campements mésolithique de plein-air : Détection, Caractérisation, Modélisation. De Ruffey-sur-Seille et Choisey (Jura), aux occupations des IX $X^{e}$ et $V I I I^{e}$ millénaires de Pont-sur-Yonne (Yonne), Thèse de doctorat, Université de Bourgogne, 316 p. 26o fig.

SÉARAF., Roncin O., 2010 : Dammartin-Marpain (Jura), Prairie du Milieu. Nouvelles données sur le peuplement mésolithique, néolithique, protohistorique et antique de la basse vallée de l'Ognon, Rapport Final d'Opération, Inrap, 2 vol., 724 p.

Souffi B., MARTi F., 2011 : Paris $15^{e}$ arrondissement, 62 rue Henry Farman. Évolution culturelle et environnementale d'un site stratifié en bord de seine, du Mésolithithique au premier âge du Fer, Rapport final d'opération, Inrap, 4 vol., 1337 p.

THÉvenin A., 2008: «Le Mésolithique ancien et moyen de la moitié nord de la France : les grandes lignes du peuplement », in FAGNART J.P. et al., p. 31-50.

Valentin B., Souffi B., Ducroce T., Fagnart J.-P., Séara F., Verjeux C. (DIR), à paraître: Palethnographie du Mésolithique: Recherches sur les habitats de plein air dans la moitié septentrionale de la France et ses marges, Actes de la table-ronde de Paris, 26-27 novembre 2010, Paris, Société préhistorique française.
Une connaissance de l'âge du Bronze transfigurée par l'archéologie préventive

Patrice Brun

Université Paris I Panthéon-Sorbonne, UMR 7041 «Archéologie des Sciences de l'Antiquité "

Cyril Marcigny

Inrap, UMR 6566 CReAAH

$\mathbf{N}$ otre connaissance de l'âge du Bronze est longtemps restée bridée par l'insuffisance des moyens de recherche sur le terrain.

Il a d'abord fallu attendre la mise en ouvre de prospections et de fouilles de grandes surfaces pour que le tableau historique et sociologique de cette période charnière se précise. Mais une autre condition a été tout aussi indispensable : la multiplication des opérations rendant possible la mise en évidence des réseaux locaux, voire régionaux, de sites en interaction. Ce double changement d'échelle de la recherche archéologique a déjà produit des résultats spectaculaires : il augure des progrès décisifs sur l'histoire de notre pays dans la longue durée.

\section{L'âge du Bronze, à la charnière de 4300 ans} longtemps mal documentés. La connaissance de la tranche de temps qui s'intercale entre les périodes paléolithique et romaine en France est longtemps restée fondée sur des découvertes de tombes et de dépôts non funéraires. À propos des sites d'habitat, on savait bien que certains avaient été fortifiés lorsque leur rempart, encore visible, avait fait l'objet de sondages permettant de le dater, mais l'architecture des bâtiments et l'organisation d'ensemble de ces établissements nous échappaient presque totalement. Quelques établissements, localisés en bord de lac et, par conséquent, mieux conservés, avaient certes fourni de précieuses indications, grâce à des fouilles d'envergure exceptionnelle, en Allemagne au Wasserburg Buchau (Reinerth, 1928), ou en Grande-Bretagne à Little Woodbury (Bersu, 1940), par exemple, mais, presque partout ailleurs, l'absence de bois conservés et l'exigüité des sondages archéologiques entretenait les préjugés primitivistes à l'égard des sociétés en question. Ainsi, malgré ces beaux exemples d'agglomérations durables, aux solides et vastes constructions en bois, terre et chaume ou joncs, on a longtemps persisté à expliquer la faiblesse des traces d'habitat par la légèreté du bâti : on envisageait des huttes construites pour de courtes occupations en raison d'un mode de vie fondamentalement nomade.

Dubitatifs sur ce point, quelques archéologues ont pu profiter du cadre légal et financier plus favorable mis en place dans leur pays pour initier des fouilles utilisant des décapages en aire ouverte sur de grandes surfaces. Des résultats spectaculaires n'ont alors pas tardé à transformer la connaissance des sites d'habitat de cette période-clé initiée par la sédentarisation graduelle qu'a rendue possible l'adoption d'une économie agro-pastorale et qui s'est achevée avec l'émergence de l'État avec ses principaux corolaires, la ville et l'écriture. L'agencement des trous des poteaux porteurs a 


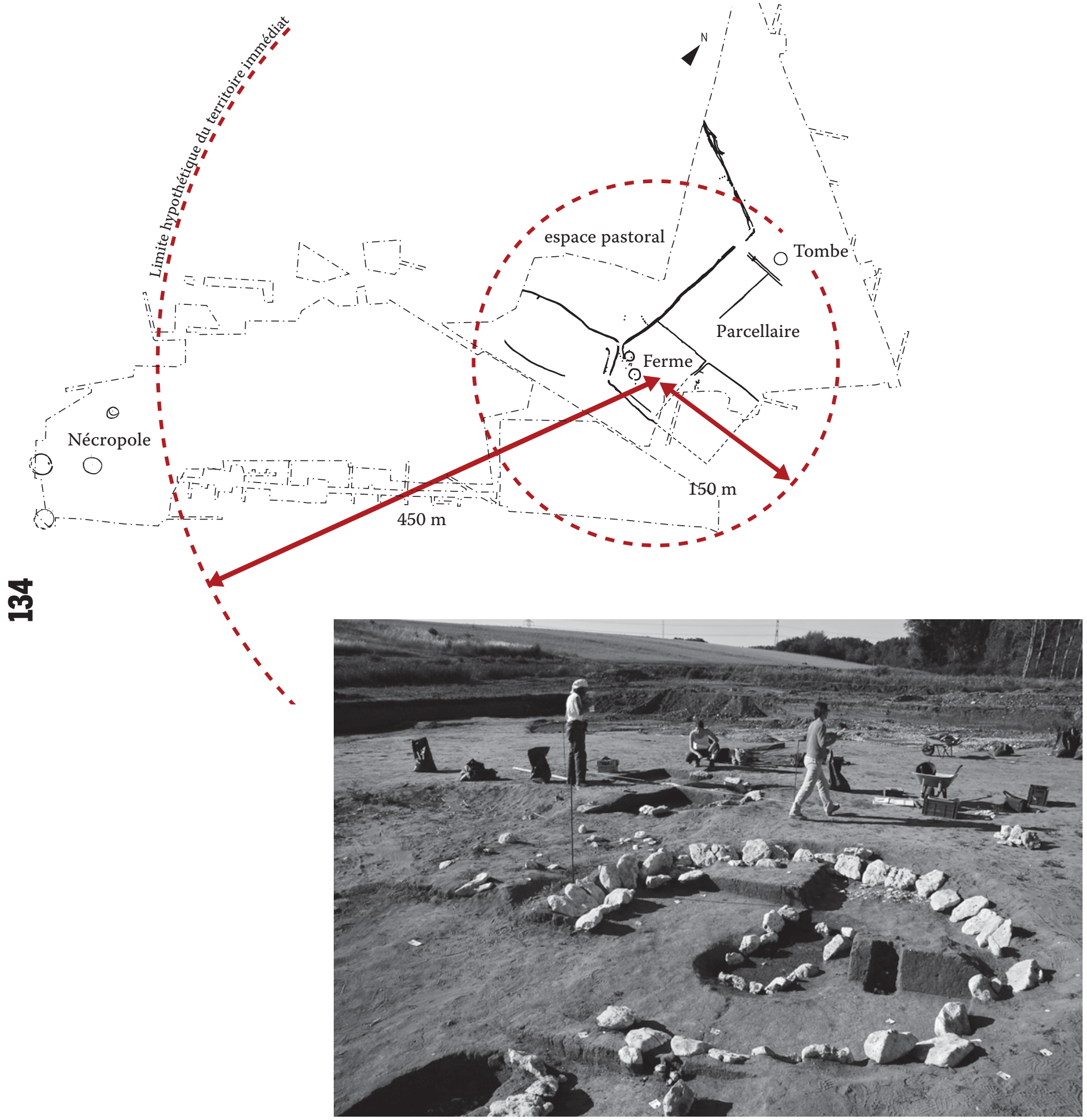

[Fig.2] Plan du site de Saint-Vigord'Ymonville (Seine-Maritime), la fouille a permis de découvrir une ferme du Bronze moyen et son espace périphérique, occupé par un parcellaire. Deux ensembles funéraires sont contemporains de cet établissement agricole: un monument situé à proximité immédiate et une petite nécropole un peu plus loin.
[Fig.3] Vue d'un des tumulus (d'environ 4 mètres de diamètre) fouillé par Hélène Froquet-Uzel (Inrap) et son équipe à Courcelles (Loiret). Sur cette petite nécropole du Bronze final, sept tumulus ont été fouillés. Le plus important mesure près de neuf mètres de diamètre ; autour gravitent six petits monuments délimités par une couronne de pierres agencées avec le plus grand soin et généralement pourvue d'une sépulture centrale. La différence de taille entre les tombes reflète probablement une

différenciation sociale qui n'est

toutefois pas marquée par des dépôts funéraires particuliers. 
en effet révélé l'existence de bâtiments parfois très vastes. De façon curieuse, toutefois, alors que, pour la période du Néolithique débutant (qui s'est achevée vers 4500 avant notre ère) et pour celle des deux derniers siècles précédant notre ère, la connaissance progressait assez régulièrement, l'habitat des quatre millénaires intermédiaires (dont l'âge du Bronze) demeurait méconnu. Il a fallu attendre un nouveau changement d'échelle des modalités d'intervention archéologique pour commencer à combler ce trou noir de la connaissance.

Le tiers de cette longue période correspond à ce que l'on appelle l'âge du Bronze, daté entre la fin du XXIII ${ }^{\mathrm{e}}$ à la fin du $\mathrm{IX}^{\mathrm{e}}$ siècle avant notre ère. Il a été individualisé $\mathrm{au} \mathrm{XIX}^{\mathrm{e}}$ siècle sur un critère technologique : l'adoption d'une métallurgie procédant de l'alliage volontaire du cuivre et de l'étain, additionné de plomb, surtout dans l'Ouest, au cours des derniers siècles de cette période. Dans les autres domaines, cette période ne se distingue pas vraiment de celles qui l'encadrent, c'est-à-dire le Néolithique et l'âge du Fer. Une métallurgie (celle du cuivre) était déjà en vigueur en France depuis quelques centaines d'années. Depuis plus longtemps encore, des écarts sociaux marqués s'affichaient dans quelques tombeaux monumentaux ou au mobilier exceptionnellement riche et dans des dépôts non funéraires de séries d'objets lithiques, mis en terre à des fins probablement votives. Symétriquement, la métallurgie du Bronze est restée majoritaire jusqu'au $\mathrm{VI}^{\mathrm{e}} \mathrm{s}$. avant notre ère pour le moins ; l'organisation sociale ne semble pas avoir beaucoup changé avant la fin du $\mathrm{I}^{\mathrm{er}}$ âge du Fer, et encore, pour une courte durée et dans quelques régions seulement; enfin, les pratiques de dépôts votifs n'ont pas cessé, même si elles sont devenues moins abondantes dans certaines zones et beaucoup plus dans d'autres, comme l'Armorique et le Languedoc. Les changements les plus significatifs pour ce que l'on appelle traditionnellement l'âge du Bronze se sont, en réalité, produits vers son milieu, entre 1600 et 1300 avant notre ère. C'est en effet à cette période seulement que le Bronze est devenu accessible dans la plupart des fermes, apparemment, pour la fabrication d'outils ayant un impact direct sur l'économie vivrière, et, par conséquent, sur la productivité agricole, la démographie, et l'intensification des flux d'échanges, donc sur l'organisation sociale globale. Se situant plutôt dans la continuité du Néolithique évolué, qui a débuté au milieu du $\mathrm{V}^{\mathrm{e}}$ millénaire, pour ses débuts (Bronze A, jusque vers 1600 avant notre ère), et annonçant les caractéristiques sociales de l'âge $\mathrm{du}$ Fer jusqu'au $\mathrm{III}^{\mathrm{e}}$ siècle avant notre ère, pour sa partie finale (du Bronze B au Hallstatt B, jusque vers 800 avant notre ère), cet âge du Bronze constitue ainsi un échantillon assez représentatif de ces 4 ooo ans trop longtemps méconnus.

Une connaissance générale des sites de cette période n'est sortie des limbes qu'à la faveur de la multiplication des opérations sur de vastes surfaces, rendues possibles par les grands progrès de la législation sur l'archéologie préventive. C'est à ce prix qu'en France, comme à l'étranger, il a été possible de réunir les observations enregistrées sur des sites suffisamment nombreux et de niveau de conservation assez varié pour compléter ou deviner les structures d'habitat manquantes ou dégradées. On s'est rendu compte que les trous de poteaux des bâtiments s'avéraient alors peu profonds, hormis ceux de constructions particulières, comme les greniers sur pilotis ou les caves et ateliers excavés. Les progrès réalisés en matière de techniques d'assemblage du bois conféraient sans doute alors aux bâtiments la solidité d'une sorte de boîte ne nécessitant qu'un faible ancrage au sol. Nous savons maintenant que ces habitations peuvent même n'avoir laissé aucune trace lorsque les montants reposaient sur des poutres, dites « sablières basses ", installées horizontalement dans un simple sillon ou bien lorsque les murs porteurs étaient en pisé. Nous savons aussi que ces établissements étaient certes plus durables que les huttes légères imaginées dans le passé de la discipline, mais moins que nos fermes et nos villages traditionnels (dont beaucoup remontent au Moyen Âge), soit guère plus d'une génération d'environ vingt-cinq ans. Nous savons enfin que l'habitat de cette période se composait surtout de fermes dispersées (Brun, 1999). Ces trois caractéristiques, une fois bien comprises, donnent enfin la possibilité de pondérer les importantes pertes d'informations et, par conséquent, de repérer les sites d'habitat qui ont si longtemps fait défaut. Force est pourtant de constater qu'elles n'ont pas encore été suffisamment intégrées : les très partiels vestiges de fermes qui apparaissent lors des diagnostics archéologiques sont encore souvent jugés sans intérêt, en tout cas ne justifiant pas une fouille. Un nombre élevé de fosses, un fossé d'enclos ou un objet exceptionnel constituent le plus souvent des conditions minimales pour prescrire une fouille digne de ce nom. Le bilan se montre, de la sorte, contrasté : d'un côté, le changement d'échelle des opérations archéologiques a bouleversé notre connaissance des sociétés protohistoriques en nous offrant les moyens de saisir les sites d'habitat qui nous échappaient, ainsi que les cimetières souvent très sobres dont nous ignorions complètement l'existence il y a peu ; de l'autre, la discrimination des sites aux vestiges modestes provoque une grave déformation du tableau sociologique recherché, en écartant de fait les documents relatifs à la paysannerie ordinaire, c'est-à-dire la très grande majorité de la population de l'âge du Bronze. Les écarts quantitatifs en qualitatifs mis en évidence entre les différentes régions administratives, à la suite de l'enquête sur les sites d'habitat de cette période (table de ronde de Bayeux, 29-30 novembre 2011), sont très significatifs à cet égard. Il est par exemple notable que dans certaines zones géographiques, pourtant très dynamiques en matière d'aménagement du territoire, très peu de prescriptions de fouilles ont été émises (voire aucune concernant l'âge du Bronze), depuis une dizaine d'années. Les découvertes ont alors bien souvent été réalisées lors de fouilles motivées par des vestiges plus spectaculaires relevant d'autres périodes. Elles résultent, pour beaucoup, de la reconnaissance 


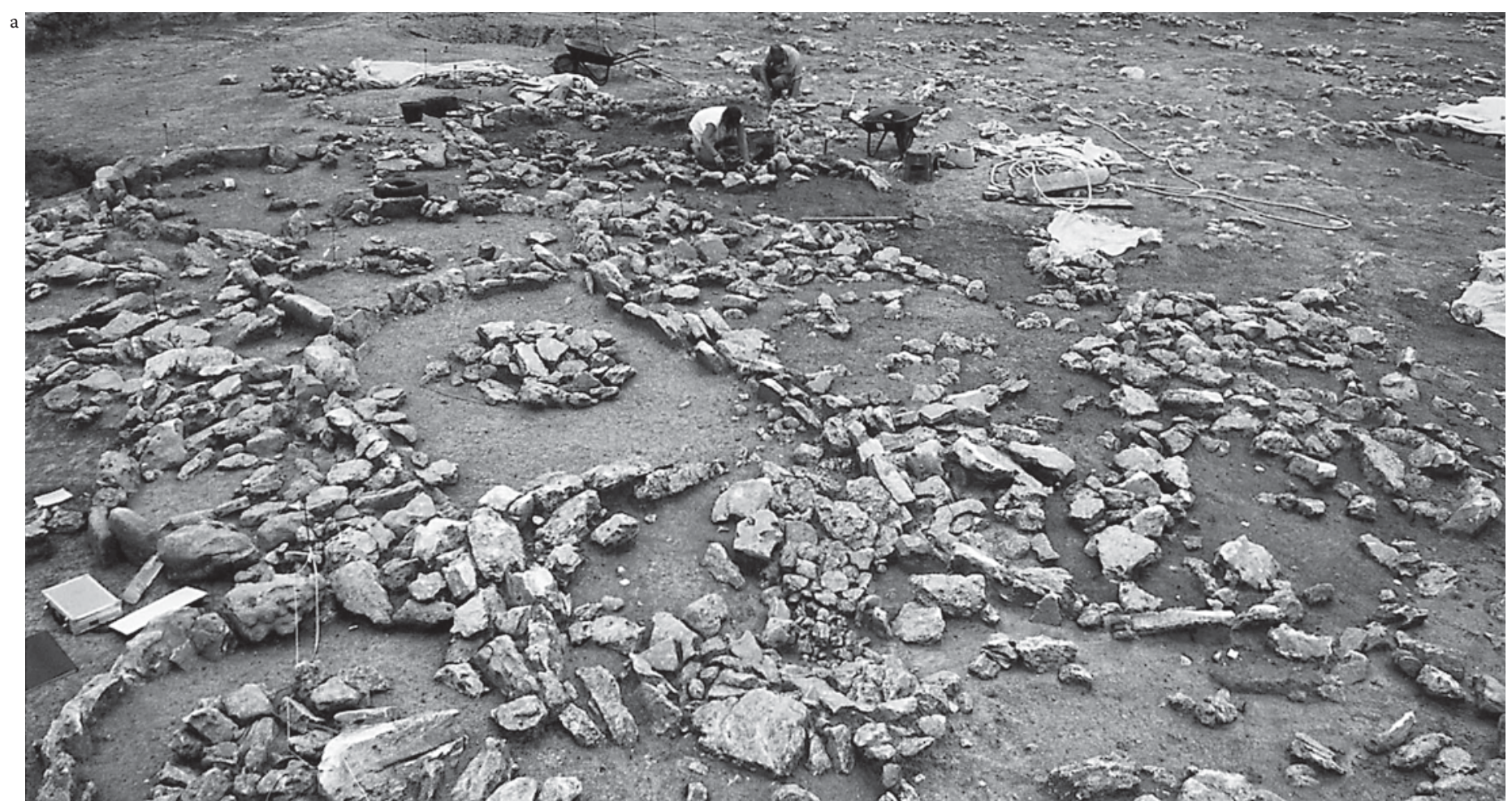

$\stackrel{\circ}{\mathscr{m}}$

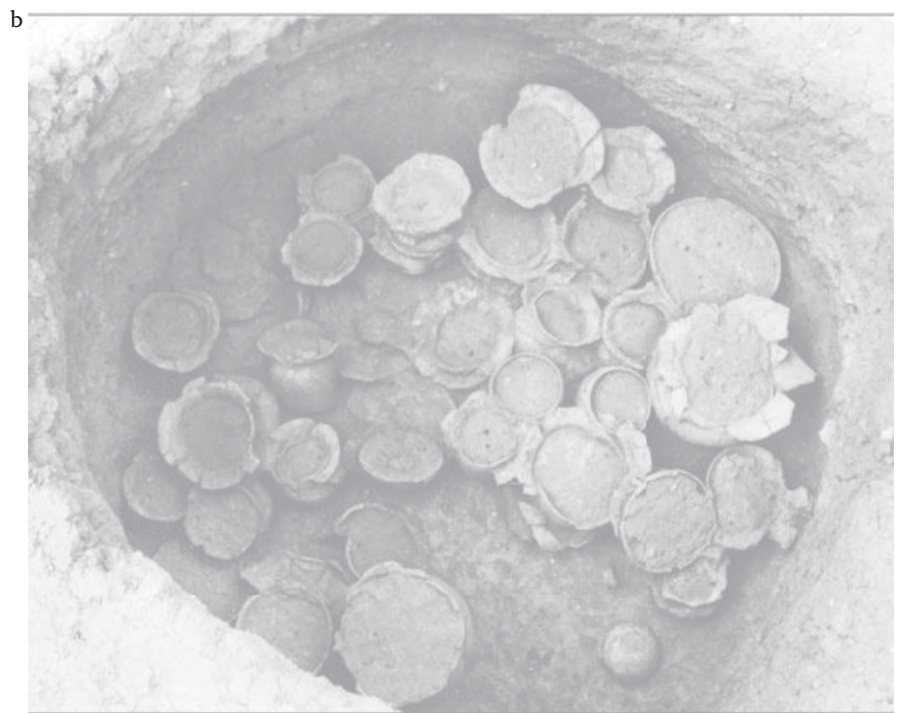

[Fig.4] La nécropole du Bronze final et du premier âge du fer de La Rouquette à Puisserguier (Hérault) en cours de fouille (responsable scientifique, Florent Mazière, Inrap). Au premie plan, on devine les dispositifs de signalisation des nombreuses sépultures constitués d'un entourage en pierre formant un plan quadrangulaire ou circulaire (a). Les

sépultures contiennent un viatique important, ici une vingtaine de vases (b). 
a posteriori, donc aléatoire, de contextes aussi bien funéraires que domestiques.

Un bouleversement des connaissances dans tous les domaines... Les résultats restent, malgré tout, importants et lèvent le voile sur tout un pan des sociétés de l'âge du Bronze, des formes de l'habitat, à une lecture plus précise de la géographie culturelle du $\mathrm{II}^{\mathrm{e}}$ millénaire, en passant par une reconnaissance des pratiques funéraires. Un des premiers apports de ce renouvellement des données a été d'affiner la géographie culturelle, de la rendre plus dynamique et de comprendre son évolution au fil du temps. Si, dans leurs grandes lignes, les découpages proposés depuis les années 1980 sur la base des contextes funéraires et des objets métalliques n'ont pas changé, avec un espace français partagé entre un bloc continental ou nord-alpin, une zone méditerranéenne et une autre atlantique, des avancées ont été effectuées sur la géométrie de ces complexes techno-culturels et sur leurs subdivisions. La découverte et l'étude d'importants corpus de mobilier céramique sont à la base de ces nouvelles lectures dynamiques, mais les autres éléments de la culture matérielle apportent aussi leur pierre à l'édifice. Parmi ces derniers, les formes de l'habitat constituent un axe de recherche important au même titre que les pratiques funéraires.

L'Ouest de la France a profité pleinement de ce renouvellement des connaissances. La géographie culturelle de ce secteur est aujourd'hui en cours de redéfinition. Longtemps cantonné à une sphère atlantique, dont on ne connaissait presque rien, si ce n'est les fameux «tumulus armoricains », il est possible aujourd'hui de distinguer, à l'intérieur de cette vaste entité aux contours flous, un groupe qualifié pour l'instant de «Manche-Mer-du-Nord» (MMN), qui s'étend de la partie orientale de la Bretagne au Nord de la France, et un groupe plus méridional, où la culture matérielle semblent davantage mêlée d'influences orientales. Au sein de ces deux groupes, les traceurs culturels sont nombreux. La céramique est, par exemple, très différente, même si un fond commun apparaît [Fig. 1]. L'habitat est aussi très dissemblable, avec des bâtiments allongés qui ne sont pas sans rappeler les constructions de l'Est de la France en Pays-de-laLoire, et des bâtiments de plan circulaire dans le groupe MMN. C'est, par ailleurs, au sein de ce dernier que vont se développer, à partir de la fin $\mathrm{du} \mathrm{III}^{\mathrm{e}}$ millénaire, des pratiques agraires qui prennent appui sur des systèmes parcellaires très élaborés, puissamment fondés dans le sol et que l'on ne retrouve pas plus au sud [Fig. 2].

Dans les autres régions, c'est la place tenue par les espaces funéraires qui apparaît comme l'élément fort. Ces espaces sont de plus en plus intégrés dans des projets d'envergure laissant une large place aux modélisations spatiales. Ces travaux, pour la plupart développés grâce à l'utilisation des systèmes d'information géographique et de modèles spatiaux-temporels ou prédictifs permettant une relecture des processus d'aménagement de l'espace, des dynamiques spatio-temporelles et de leurs trajectoires évolutives, ont mis en évidence le rôle de géosymbole tenu par les ensembles funéraires [Fig. 3]. Ces marqueurs de l'organisation territoriale fonctionnaient en réseau au même titre que l'habitat. Ils étaient manifestement empreints d'une forte charge symbolique exprimant une forme d'appropriation de l'espace qui participait activement à la construction des paysages. Ainsi, dans le Sud de la France, de très grandes nécropoles, dont certaines intégrant parfois plusieurs centaines d'individus, forment de vastes « villes des morts », déconnectées des habitats et occupant des lieux particuliers comme des interfluves ou des confluents [Fig.4].

\section{... mais une difficulté persistante à saisir les} réseaux sociaux dans toutes leurs dimensions. Les opérations réalisées sur de très grandes surfaces ont ainsi permis d'étudier des établissements dans la totalité de leurs vestiges préservés. Les sites de l'âge du Bronze répertoriés de la sorte s'avèrent, en général, beaucoup plus nombreux et divers que ce que laissaient prévoir les connaissances et les prospections préalables. Les sites domestiques se révèlent présents sur presque toutes les opérations, bien que sous des formes très érodées. Les tombes, dispersées ou en petits groupes, sont très sobres et ne subsistent, elles aussi, qu'à l'état résiduel, surtout lorsqu'il s'agit de crémations, sauf dans le Sud-Ouest du pays où une plus large fraction de la population avait accès au cimetière communautaire. Pour divers et partiels qu'ils soient, les résultats obtenus commencent, en somme, à nous révéler la trame du peuplement de cette période. Les établissements sont surtout des fermes, parfois fortes, des hameaux dans une moindre mesure, et des villages plus rarement encore, fortifiés ou non.

Un autre apport, plus spécifique encore, des fouilles de très grandes surfaces, est de mettre en évidence plusieurs sites classables dans une même étape chronologique. Le degré de précision de notre système typochronologique et les possibilités de datation absolue ne permettent toujours pas, malheureusement, d'établir leur contemporanéité stricte. Nous disposons pourtant de bons indices suggérant que les petits établissements de l'âge du Bronze étaient abandonnés et reconstruits à une certaine distance tous les vingt-cinq ans en moyenne, sur une même aire d'approvisionnement local, appelée un « finage ». Nous cherchons désormais à mieux connaître les modalités de ces déplacements et la composition des réseaux locaux dans lesquels ces finages se trouvaient intégrés. Les établissements dispersés appartenaient nécessairement, en effet, à des réseaux de sociabilité qui ne peuvent être saisis qu'à une échelle élargie (Brun et al., 2006).

Une échelle de fouille macroscopique permet enfin d'analyser des réseaux locaux dans leur dynamique évolutive. Elle révèle les changements de formes et de rythmes directement perceptibles dans la succession des formes d'occupation des paysages et d'exploitation agropastorale. Nous observons 


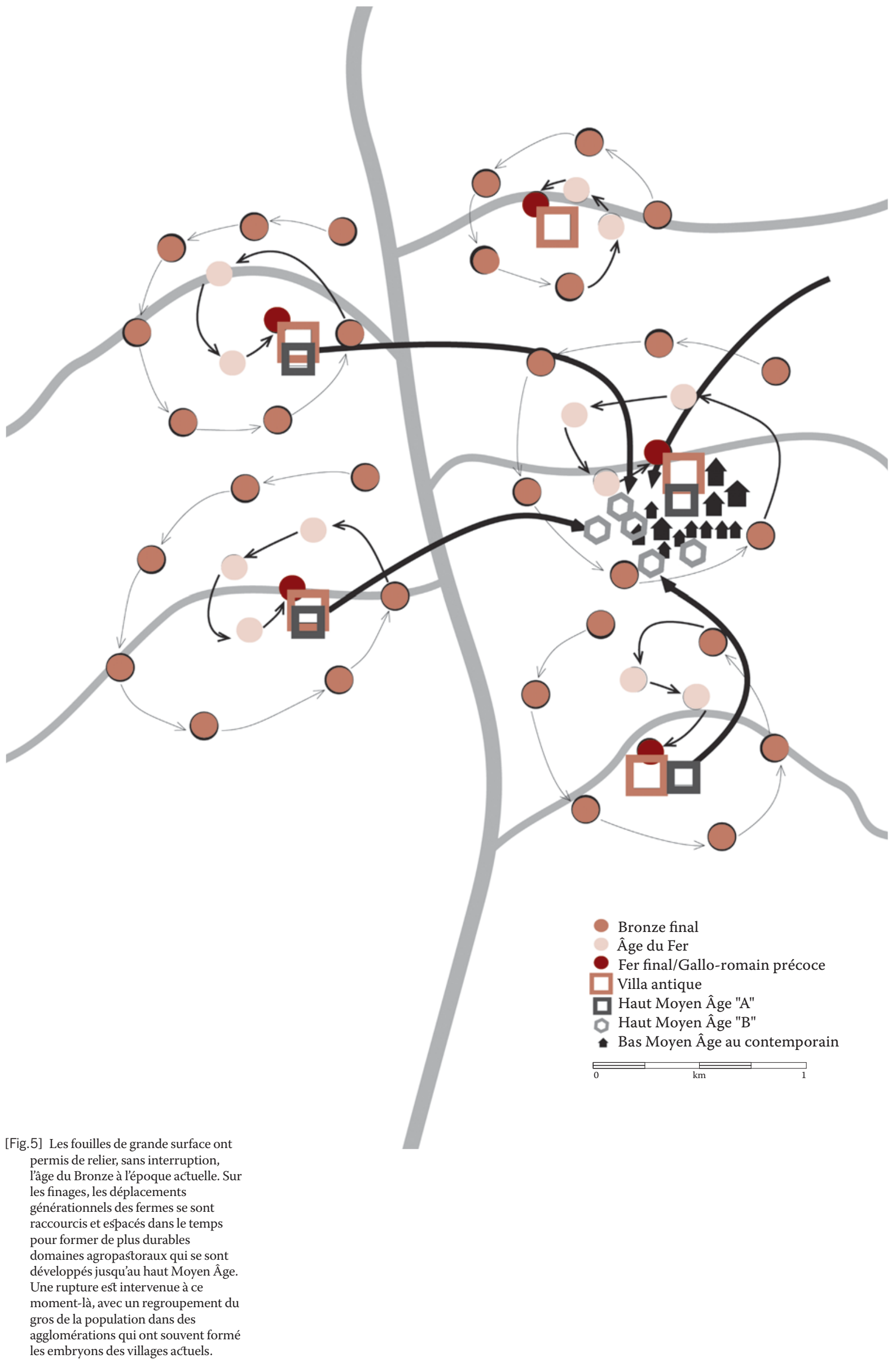


des oscillations dans la densité des établissements, avec, parfois même, par endroits, des disparitions momentanées. Les périodes de déprise doivent, bien sûr, être enregistrées et sérieusement vérifiées afin de fonder solidement l'interprétation historique qui est la finalité de notre discipline.

Les fouilles de grandes surfaces nous apprennent d'ores et déjà qu'à partir du Bronze moyen $\left(\mathrm{XVI}^{\mathrm{e}} \mathrm{s}\right.$. avant notre ère) des finages tendent à se pérenniser. Sur ces aires d'approvisionnement, il semble que les déplacements générationnels des fermes se soient graduellement raccourcis dans leur finage et espacés dans le temps. Cette évolution ne s'est pas produite de manière linéaire, mais dans une succession de tentatives plus ou moins durables et variables d'une région à une autre. Lorsqu'il devenait plus durable, le domaine agricole tendait à développer ses infrastructures sur l'espace qu'il exploitait - palissades de prestige et de protection, limites de champs, parcellaires et aménagements de voies de circulation -, afin de rationaliser et d'intensifier la production vivrière. Certaines régularités métrologiques sont même discernables dans le parcellaire, ce qui pose la question, aux conséquences majeures, des taxes foncières et du régime de propriété. Nous voyons ces systèmes de champs se développer jusqu’à l'époque gallo-romaine, voire le haut Moyen Âge. Une rupture paraît intervenir à ce moment-là, avec un regroupement de la population issue d'un certain nombre de domaines agricoles. Naissent alors des agglomérations qui forment souvent les embryons des villages actuels (Blaising, 2002) [Fig. 5]. Ces enchaînements, que seules des fouilles de grande surface sont aptes à mettre en évidence, relient, pour la première fois sans interruption, lâge du Bronze à l'époque actuelle. La mise en lumière de cette tendance lourde s'avère d'une grande portée historique potentielle. Il reste à en préciser la séquence globale, à distinguer les différences spatiales selon les spécificités, les traditions culturelles et les formes d'organisations sociétales.

Les enjeux de ce nouveau changement d'échelle dépassent d'ailleurs la question de ces configurations spatiales, tellement cruciales pour les enquêtes de ce type, car seules révélatrices des organisations sociétales recherchées. Ils touchent, en effet, aux valeurs culturelles propres à l'Europe tempérée humide qu'implique le caractère foncièrement dispersé de l'habitat pendant une aussi longue période, c'est-à-dire la Protohistoire au sens large, de la néolithisation à l'urbanisation. Ces valeurs émanaient, pour partie, de la structure familiale et lignagère induite par cette dispersion et cette relative mobilité. Il en résultait, inévitablement, des particularités touchant des aspects sociaux fondamentaux puisque, dans ce cas, l'appartenance au groupe ne repose évidemment pas sur la résidence, mais sur la parenté. Cela implique des formes d'échanges, de communications, d'expressions, de solidarités, de mobilisations collectives, de pouvoirs et de perceptions de l'environnement naturel et social bien particulières. Ces structures profondes, qui ont imprégné, pendant au moins trois mille ans, les mentalités et les formes d'organisation sociale dans cette Europe tempérée humide, ont vraisemblablement conditionné son histoire ultérieure, caractérisée par une persistante division politique et une mise en échec récurrente des pouvoirs forts de type impérial. Le contraste est, en effet, très fort entre les durables empires asiatiques (égyptiens, mésopotamiens, perses, indo-pakistanais, chinois) ou américains (andins et méso-américains) et les éphémères formations européennes qualifiées d'empires, Rome comprise. Nous avons désormais les moyens de montrer que notre histoire ancienne, bien que fondée presque exclusivement sur des sources matérielles, se trouve en mesure d'éclairer le passé récent et l'actualité par la prise en compte des réseaux. La ferme en elle-même ne nous importe vraiment qu'au travers du réseau local qu'elle contribuait à animer et dont les caractéristiques de localisation relative, de nombre, de fonction et de hiérarchie des établissements intégrés, nous permettent d'accéder à des interprétations sociales et culturelles infiniment plus riches.

Bersu G., 1940: « Excavations at Little Woodbury, Wiltshire. Part I: the settlement as recorded by excavation ", Proceedings of the Prehistoric Society, 6, p. 30-311.

Blaising J.-M., 2002 : « Les formes de l'occupation du sol des âges des métaux à nos jours en vallée de Moselle », Medieval Europe Basel, vol. 1, p. 78-83.

BRUN P., 1999: «L'habitat: prisme déformant de la société protohistorique », in Braemer F., Cleuziou S., Coudart A. (Dir.), Habitat et société, Actes des XIX ${ }^{\mathrm{e}}$ Rencontres Internationales d'Archéologie et d'Histoire d'Antibes, Antibes, éditions APDCA, p. 339-352

BRUn P., MARCigny C., VANMoerkerke J. (DIR.), 2006 : Une archéologie des réseaux locaux: quelles surfaces étudier pour quelle représentativité ?, Les Nouvelles de l'Archéologie, $\mathrm{n}^{\circ}$ 104-105, Paris, Errance.

Reinerth H., 1928 : Die Wasserburg Buchau, Augsbourg, B. Filser.

\section{De la détection des structures fugaces à la reconnaissance d'un système funéraire: les fosses à résidus de combustion de lâge du Bronze}

Isabelle Le Goff

Inrap, UMR 7041 «Archéologies et Sciences de l'Antiquité » Ghislaine Billand

Inrap, UMR 8164 « Histoire, Archéologie, Littératures des Mondes Anciens. Institut de Papyrologie et d'Égyptologie de Lille »

$\mathrm{L}$ es archéologues, bien que leur discipline ait déjà une longue histoire, découvrent encore des formes inédites de structures et intègrent toujours de nouveaux outils techniques ou conceptuels. Par ailleurs, le rapport à la réalité du passé se modifie en fonction de la lecture que l'on sait en faire dans le présent. Cela implique, comme le propose Laurent Olivier, que la structure archéologique en tant que « (...) lieu du passé n'est pas le passé lui-même mais bien le présent et lui seul.» (Olivier, 2004). Les pratiques funéraires dont il sera question ici, celles qui caractérisent en particulier la fin de l'âge du Bronze dans le Nord de la France, sont un exemple de l'évolution de notre perception d'une structure archéologique qui laisse 